\title{
Explosion of very massive stars and the origin of intermediate mass black holes
}

\author{
Sachiko Tsuruta, ${ }^{1}$ Takuya Ohkubo, ${ }^{2}$ Hideyuki Umeda, ${ }^{2}$ \\ Keiichi Maeda, ${ }^{3}$ Ken'ichi Nomoto, ${ }^{2,4}$ Tomoharu Suzuki ${ }^{2}$ \\ and Martin J. Rees ${ }^{5}$ \\ ${ }^{1}$ Department of Physics, Montana State University, Bozeman, MT 59717, USA \\ email: uphst@gemini.msu.montana.edu \\ ${ }^{2}$ Department of Astronomy, Univ. of Tokyo, Hongo, Bunkyo-ku, Tokyo, Japan \\ ${ }^{3}$ Department of Earth Sci. and Astron., Univ. of Tokyo, Komaba, Meguro-ku, Tokyo, Japan \\ ${ }^{4}$ Research Center for the Early Universe, Univ. of Tokyo, Hongo, Bunkyo-ku, Tokyo, Japan \\ ${ }^{5}$ Institute of Astronomy, University of Cambridge, Madingley Road, Cambridge CB3 0HA, UK \\ email: ohkubo@astron.s.u-tokyo.ac.jp, umeda@astron.s.u-tokyo.ac.jp, \\ nomoto@astron.s.u-tokyo.ac.jp, suzuki@astron.s.u-tokyo.ac.jp, maeda@esa.c.u-tokyo.ac.jp, \\ mjr@ast.cam.ac.uk
}

\begin{abstract}
We calculate evolution, collapse, explosion, and nucleosynthesis of Population III very massive stars with $500 M_{\odot}$ and $1000 M_{\odot}$. It was found that both $500 M_{\odot}$ and $1000 M_{\odot}$ models enter the region of pair-instability but continue to undergo core collapse to black holes. For moderately aspherical explosions, the patterns of nucleosynthesis match the observational data of intergalactic and intercluster medium and hot gases in M82, better than models involving hypernovae and pair instability supernovae.

Our results suggest that explosions of Population III core-collapse very massive stars contribute significantly to the chemical evolution of gases in clusters of galaxies. The final black hole masses are about $500 M_{\odot}$ for our most massive $1000 M_{\odot}$ models. This result may support the view that Population III very massive stars are responsible for the origin of intermediate mass black holes which were recently reported to be discovered.
\end{abstract}

\section{Introduction}

One of the most interesting challenges in astronomy is to investigate the mass and properties of first generation 'Population III (Pop III)' stars, and how various elements have been synthesized in the early universe. Just after the Big Bang these elements were mostly only $\mathrm{H}, \mathrm{He}$ and a small amount of light elements ( $\mathrm{Li}, \mathrm{Be}, \mathrm{B}$, etc). Heavier elements, such as $\mathrm{C}, \mathrm{O}, \mathrm{Ne}, \mathrm{Mg}$, $\mathrm{Si}$ and $\mathrm{Fe}$, were synthesized during the evolution of later generation stars, and massive stars exploded as supernovae ( $\mathrm{SNe}$ ), releasing heavy elements into space.

The fate of zero-metalicity Pop III stars are: Stars lighter than $8 M_{\odot}$ form white dwarfs; those with $8 M_{\odot}-130 M_{\odot}$ generally explode as the core-collapse supernovae leaving neutron stars or black holes as compact remnants; stars with $130 M_{\odot}-300 M_{\odot}$ disrupt completely as the pair-instability supernovae (PISN) with no compact remnants left; stars with $300 M_{\odot}$ to $\sim 10^{5} M_{\odot}$ (without rotation) collapse to black holes with no explosion; and those over $10^{5} M_{\odot}$ collapse directly to black holes before reaching the main-sequence. (See, e.g., Fryer, Woosley \& Heger 2001)

It has been suggested that the initial mass function (IMF) of Pop III first stars is probably more massive than, e.g., the Salpeter mass function (e.g., Abel, Bryan \& 
Norman 2000; Bromm, Coppi \& Larson 2002; Qian, Sargent \& Wasserburg 2002). Numerical simulations by, e.g. Bromm \& Loeb (2004) indicate that the maximum mass of Pop III stars to be formed will be $\sim 300 M_{\odot}-500 M_{\odot}$. Omukai \& Palla $(2003)$, however, point out that under certain conditions very massive stars much heavier than $300 M_{\odot}$ can be formed in the zero-metalicity environment. Another scenario for the formation of very massive stars for any metallicity has been presented by Ebisuzaki et al. (2001) and Portegies Zwart et al. (2004), where very massive stars are formed by merging of less massive stars in the environment of very dense star clusters.

In the present paper, we call the stars with $M=130 M_{\odot}-10^{5} M_{\odot}$ 'Very-Massive Stars (VMSs)'. Among VMSs we define $M>300 M_{\odot}$ stars as 'Core-Collapse Very-Massive Stars (CVMSs)', in order to clarify the distinction between the PISN mass range and the core-collapse range. Here we focus on CVMSs, and deal with $500 M_{\odot}$ and $1000 M_{\odot}$ models.

Stellar mass black holes $\left(\sim 10 M_{\odot}\right)$ are formed as the central compact remnants of ordinary massive $\left(25-130 M_{\odot}\right)$ stars at the end of their evolution. Supermassive black holes $(\mathrm{SMBHs})\left(\sim 10^{5}-10^{9} M_{\odot}\right)$ are now known to exist in the center of almost all galaxies (e.g., Kormendy \& Richstone 1995), and there are several scenarios for their formation (e.g., Rees's contribution to this volume). As to intermediate mass black holes (IMBHs) with $\sim 5 \times 10^{(2-4)} M_{\odot}$, there is a strong possibility that some of these stars have been discovered as ULX (see, e.g., contribution to this volume by Makishima). The question of whether CVMSs $\left(\sim 300 M_{\odot}-10^{5} M_{\odot}\right)$ actually existed is of great importance, for instance, to understand the origin of IMBHs.

Also, if CVMSs existed and if they were rotating, they might have released a large amount of heavy elements into space by mass loss and/or supernova explosions. If so, they might have significantly contributed to the early galactic chemical evolution because they could be the source of reionization of intergalactic $\mathrm{H}$ and He (e.g., Gnedin \& Ostriker 1997; Venkatesan et al. 2003). The reionization of intergalactic He has traditionally been attributed to quasars. However, according to the results of the the Wilkinson Microwave Anisotropy Probe (WMAP) observation in 2003, reionization in the universe took place as early as 0.2-0.3 billion years after the Big Bang (redshift $\mathrm{z}>\sim 20$; Kogut et al. 2003). Then these Pop III CVMSs might provide a better alternative channel which could operate at redshifts higher than what is assumed for quasars (Bromm, Kudritzki \& Loeb 2001).

\section{Our Recent Research}

\subsection{Our Models}

Motivated by the backgrounds as outlined in the previous section, we calculated evolution, core-collapse, explosion, and nucleosynthesis of Pop. III CVMSs (Ohkubo et al. 2006, hereafter O06). We start our evolutionary calculations by assuming that the stars have $500 M_{\odot}$ and $1000 M_{\odot}$ with zero-metalicity on the pre-main sequence. Without rotation these stars are expected to form black holes directly with no explosion at the end of evolution.

However, if the star is rotating the whole star probably will not become a black hole at once, but form an accretion disk around the central remnant. After forming an accretion disk, jet-like explosions may occur by extracting energy from the accretion disk and/or the black hole itself. Therefore, in our explosion and collapse calculations we adopt a twodimensional approach including accretion along the equatorial direction and jets toward the polar direction. 
To calculate pre-supernova evolution we adopt the stellar evolution code constructed by Umeda \& Nomoto (2002) based on the Henyey method. We include 51 isotopes up to Si until He burning ends, and 240 up to Ge afterwards. Our evolutionary calculations are carried out from the pre-main sequence up to the iron-core collapse where the central density reaches as high as $2 \times 10^{10} \mathrm{gcm}^{-3}$.

When the temperature reaches $5 \times 10^{9} \mathrm{~K}$, where 'nuclear statistical equilibrium' is realized, the abundance of each isotope is determined for a given set of density, temperature, and the number of electrons per nucleon. Our CVMSs are assumed to explode in a form of bipolar jets, and we explored the required constraints. We adopted two types of models: Case (A) where almost all the energy of the jet is given as kinetic energy, and Case (B) where almost all the energy is given as thermal energy. Case (A) jets are better collimated, while Case (B) jets are only mildly aspherical. See O06 for further details.

\subsection{Results and Discussion}

Although our $500 M_{\odot}$ and $1000 M_{\odot}$ stars pass through the region of electron-positron pair-instability, both stars proceed to iron-core collapse, unlike a $300 M_{\odot}$ star.

The region which experiences explosive silicon burning to produce iron-peak elements is more than $20 \%$ of the total mass, much larger than those of ordinary massive stars such as a $25 M_{\odot}$ star. Note that for the metal-free $25 M_{\odot}$ star model, this fraction is less than $10 \%$ (Umeda \& Nomoto 2002). This is because for the $500 M_{\odot}$ and $1000 M_{\odot}$ models the density and temperature distributions are much flatter than those of $25 M_{\odot}$ stars.

Typical explosion energy is of the order of $10^{54} \mathrm{erg}$ for $1000 M_{\odot}$ models for the parameter ranges in this study.

Masses of the remnant black holes we found are $\sim 500 M_{\odot}$ for the $1000 M_{\odot}$ star models. Note that such a black hole mass is very similar to those of IMBHs, e.g., a claimed $\sim 700 M_{\odot}$ black hole in M82. It is quite possible that CVMSs could be the progenitors of IMBHs.

Nucleosynthesis yields of CVMSs have similar patterns of $[\alpha / \mathrm{Fe}]$ to the observed abundance patterns of both intercluster medium (ICM) and hot gases in M82 if the contribution of the jet is small (Case B). Specifically, for Case B small ratios of $[\mathrm{O} / \mathrm{Fe}]$ and $[\mathrm{Ne} / \mathrm{Fe}]$ combined with large $[\mathrm{Mg} / \mathrm{Fe}],[\mathrm{Si} / \mathrm{Fe}]$ and $[\mathrm{S} / \mathrm{Fe}]$ (i.e. large $[(\mathrm{Mg}, \mathrm{Si}, \mathrm{S}) / \mathrm{O}]$ ) are generally more consistent with these observational data of ICM, than those of hypernovae and PISNe.

[C/Si] of our CVMS models is compatible with that of intergalactic medium (IGM) at high redshift $(z=5)$, which is sufficiently higher and thus better than those of PISNe.

For Fe-peak elements, the main feature of the yields of our Case B CVMSs is that $[\mathrm{Cr} / \mathrm{Fe}]$ and $[\mathrm{Mn} / \mathrm{Fe}]$ are small while $[\mathrm{Co} / \mathrm{Fe}]$ and $[\mathrm{Zn} / \mathrm{Fe}]$ are large. This is consistent with the observed ratios in the extremely metal-poor (EMP) stars. The over-solar ratios of some $\alpha$-elements, such as $[\mathrm{Mg} / \mathrm{Fe}]$ and $[\mathrm{Si} / \mathrm{Fe}]$, are also consistent with EMP stars.

We estimated the ionization efficiency of our CVMSs. It was found that the number of ionizing photons per baryon in the universe, generated in association with the IGM metalicity $Z_{\mathrm{IGM}} \sim 10^{-4}$, is $N_{\mathrm{Lyc}} / N_{\mathrm{b}} \sim 150$, and so CVMSs can contribute significantly to reionization of IGM in the early epoch. Here we emphasize that our current result for CVMSs is contributed from the mass range with $\sim 300 M_{\odot}-1000 M_{\odot}$ and thus the PISN (pair instability supernova) range is not included. On the other hand, Venkatesan \& Truran $(2003)$ give $N_{\text {Lyc }} / N_{\mathrm{b}} \sim 10$ for $Z_{\text {IGM }} \sim 10^{-4}$ for the mass range $\sim 100 M_{\odot}-1000 M_{\odot}$. This outcome therefore reflects the large contribution of PISNe to metal enrichment. Note that in their models CVMSs do not explode.

The relation between reionization and metal enrichment of IGM becomes clearer if we solve the equation for $N_{\mathrm{Lyc}} / N_{\mathrm{b}}$ (Eqn. 2 of Venkatesan \& Truran 2003) for a given value of 
$Z_{\mathrm{IGM}}$. For a $1000 M_{\odot}$ star, $Z / Z_{\odot} \sim 10^{-3.4}$ and $10^{-4.4}$ for the required number of ionizing photons per baryon 10 and 1 , respectively. This is about one order of magnitude smaller than the case for the mass range $100 M_{\odot}-1000 M_{\odot}$ (mainly contribution from PISNe). The difference between CVMSs and PISNe is larger if we consider the enrichment of iron. The $260 M_{\odot}$ PISN of Heger \& Woosley (2002) gives $Z_{\mathrm{Fe}} / Z_{\mathrm{Fe}, \odot} \sim 10^{-2}-10^{-3}$, while our $1000 M_{\odot}$ star gives $\sim 10^{-3.2}-10^{-4.2}$.

A main critique against the existence of PISNe comes from the fact that we do not see the abundance patterns of PISNe in EMP stars (Tumlinson et al. 2004). The EMP abundances were suggested to be accounted for by hypernovae or faint supernovae from less massive stars of $<\sim 100 M_{\odot}$ (Umeda \& Nomoto 2003). However, the apparent lack of evidence of VMSs by no means contradicts the existence of CVMSs at earlier epochs, if a majority of first stars in the earlier epoch has masses $>\sim 300 M_{\odot}$. First, in this case PISNe from stars of $<\sim 300 M_{\odot}$ will be just a minor fraction, which explains well the lack of the signature of PISNe. Second, $Z / Z_{\odot}$ expected from our CVMSs is smaller than PISNe. Namely, the metal enrichment by CVMSs might be finished before ordinary core-collapse SNe become dominant.

Here we have shown that the yields of our CVMSs can reproduce the abundance of IGM. In our scenario CVMSs are first formed in pre-galactic mini halos, and then subsequently ordinary core-collapse SNe took place in the galactic halo.

\section{Acknowledgements}

We would like to thank useful comments given by some colleagues in the audience, especially Dr. Portegies Zwart.

\section{References}

Abel, T., Bryan, G. L. \& Norman, M. L. 2000, ApJ, 540, 39

Bromm, V., Coppi, P. S. \& Larson, R. B. 2002, ApJ, 564, 23

Bromm, V., Kudritzki, R. P. \& Loeb, A. 2001, ApJ, 552, 464

Bromm, V. \& Loeb, A. 2004, NewA, 9, 353

Ebisuzaki, T., Makino, J., Tsuru, T. G., Funato, Y., Portegies, Zwart, S., Hut, P. et al. 2001, ApJ, 562, L19

Fryer, C. L., Woosley, S. E. \& Heger, A. 2001, ApJ, 550, 372

Gnedin, N. Y. \& Ostriker, J. P. 1997, ApJ, 486, 581

Heger, A. \& Woosley, S. E. 2002, ApJ, 567, 532

Kogut, A., Spergel, D. N., Barnes, C., Bennett, C. L., Halpern, M. \& Hinshaw, G. et al. 2003, ApJS, 148, 161

Kormendy, J. \& Richstone, D. 1995, ARA\&A, 33, 581

Ohkubo, T., Umeda, H., Maeda, K., Nomoto, K., Suzuki, T., Tsuruta, S. et al. 2006, ApJ, 645, 1352 (O06)

Omukai, K. \& Palla, F. 2003, ApJ, 589, 677

Portegies, Zwart, S. F., Baumgardt, H., Hut, P., Makino, J. \& McMillan, S. L. W. 2004, Nature, 428,724

Qian, Y.-Z, Sargent, W. L. W. \& Wasserburg, G. J. 2002, ApJ, 588, 1099

Tumlinson, J., Venkatesen, A. \& Shull, J. M. 2004, ApJ, 612, 602

Umeda, H. \& Nomoto, K. 2002, ApJ, 565, 385

Umeda, H. \& Nomoto, K. 2003, Nature, 422, 871

Venkatesan, A., Tumlinson, J. \& Shull J. M. 2003, ApJ, 584, 621

Venkatesan, A. \& Truran, J. W. 2003, ApJ, 594, L1

Alexander Zakharov: 1. How did you choose initial conditions for 2-D explosion calculations using 1-D evolution calculations? 2. Have you solved the so-called "energetic problem" to explain supernova explosion? 
SAchiko Tsuruta: Answering these questions requires a complicated exploration; a referee of our ApJ paper asked exactly the same questions and our answers are given in detail there (Ohkubo et al., ApJ, July 10, 2006). So, please, read this paper for the answers to your questions. The energy source is accretion and jets.

Simon Portegies Zwart: Comment: it is very interesting that you require rapid rotation in the pre-SN star. This is a natural consequence of the collision runaway scenario to make $\sim 1000 M_{\odot}$ stars in young and dense star clusters.

SACHIKo Tsuruta: In our next work we are hoping to include binary merging during the stellar evolution stages.

DANY VANBEVEREN: Very massive stars have very large stellar wind mass-loss rates; also low- $Z$ very massive stars. What is the mass-loss rate prescription that you are using?

Sachiko Tsuruta: At the next stage we will include mass loss, though we have not included it in our first approximation. Our guess is that large mass star (e.g. $\sim 5000 M_{\odot}$ ) are needed to produce a $500 M_{\odot}$ black hole. We discussed this problem in Ohkubo et al. (ApJ, July 10, 2006). 


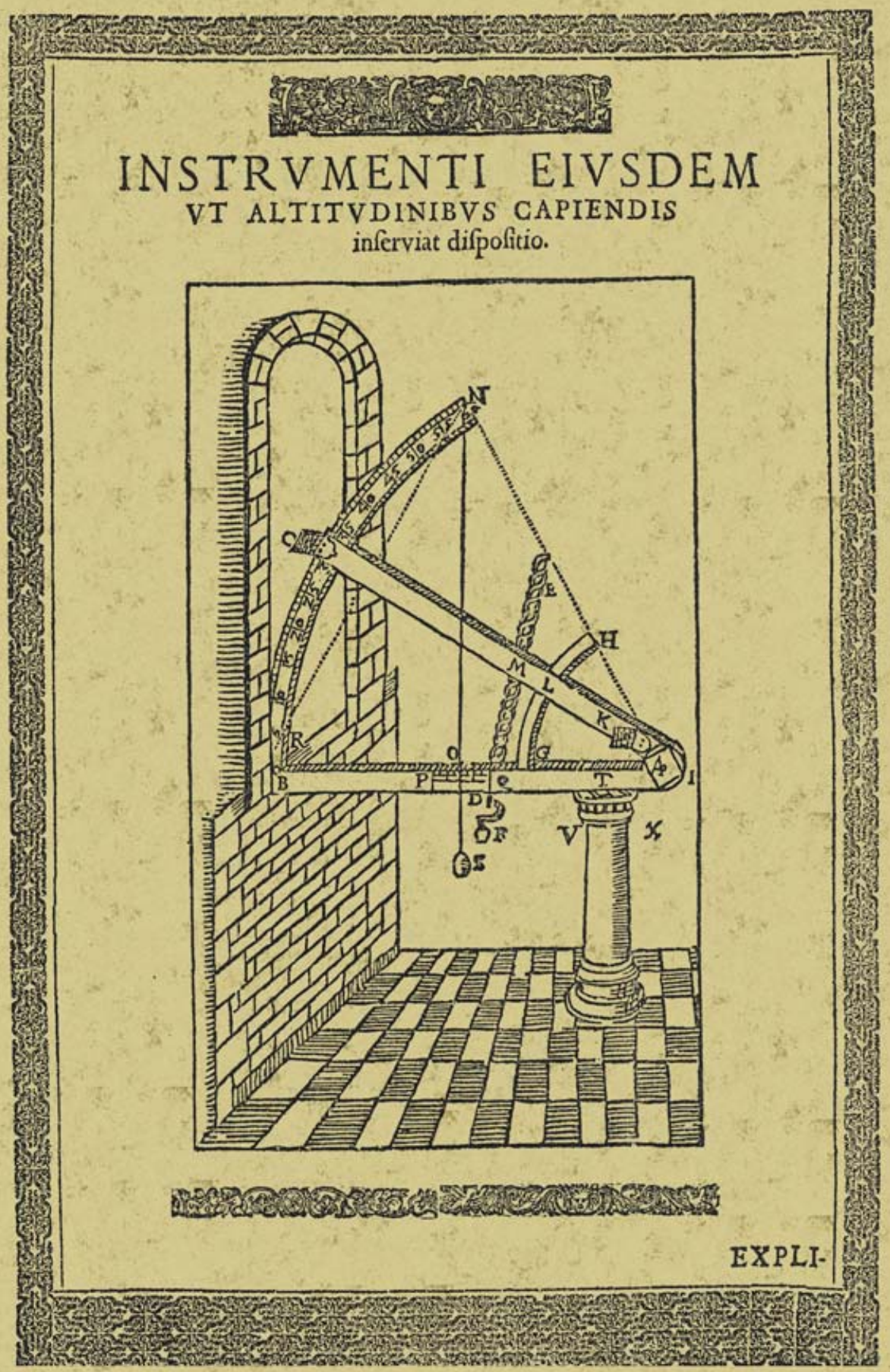

